\title{
Genetic Networks with SUM Regulatory Functions: Characterizing the Equilibrium Points
}

\author{
Graziano Chesi \\ Department of Electrical and Electronic Engineering \\ University of Hong Kong \\ Hong Kong, China \\ http: //www. eee.hku.hk/ Chesi
}

\begin{abstract}
Genetic networks with SUM regulatory functions are a fundamental class of models studied in systems biology. A primary issue for these models consists of establishing the number of the equilibrium points and their location. Unfortunately, this is a difficult problem, indeed existing methods very often do not allow one to solve it. This paper proposes a study of this problem, and describes an approach that exploits the properties of SUM regulatory functions in order to correctly characterize these points of interest. This is verified by some numerical examples, which illustrate the proposed solution and show the advantages with respect to existing methods.
\end{abstract}

Index Terms-Genetic network, SUM regulatory function, Equilibrium point, Nonlinear system.

\section{INTRODUCTION}

A fundamental research area of systems biology is represented by genetic regulatory networks, which explain the interactions between genes and proteins, see for instance [1][5]. In fact, these interactions allow to form complex systems, able to perform complicated biological functions. Two types of genetic regulatory network models are mainly considered. The first type, known as Boolean model (or discrete model), expresses the activity of each gene in one of two states (ON or OFF) and describes the state of a gene by a Boolean function. The second type, known as differential equation model (or continuous model), uses the concentrations of gene products (such as mRNAs and proteins) as variables. See for example [6]-[10] and references therein for a wider categorization of genetic regulatory networks models.

In genetic regulatory networks described via differential equation models, a key problem is to establish the number of the equilibrium points and their location. An equilibrium point represents the amounts of concentrations for which no regulation process is activated, and is hence a steady-state solution of the system of differential equations. This problem is motivated by the fact that the knowledge of the equilibrium points is required in several investigations, such as stability and disturbance rejection.

Unfortunately, it is well-known that the equilibrium points of genetic regulatory networks cannot be easily calculated. Indeed, this problem is presently a difficult one as genetic regulatory networks contain nonlinear functions, and hence the equilibrium points are the solutions of a system of nonlinear equations. In fact, existing methods allow one to characterize the equilibrium points of genetic regulatory networks only in the case of polynomial equations, which however can be addressed only for small size systems. See for instance [11] [14] and references therein. Moreover, in the case of nonpolynomial equations, no existing method allows one to obtain this characterization, as there is no strategy able to guarantee to find all solutions of a system of non-polynomial equations.

This paper describes a possible solution for establishing the number of the equilibrium points and their location in genetic networks with SUM regulatory functions. These networks are described by differential equation models where the dynamics of each concentration is expressed by a function containing two parts: a linear part which defines the natural decay rate of the concentration itself, and a nonlinear part which defines the influence on this concentration by all the other ones and which is expressed as a linear combination of saturation functions such as the Hill functions, see for instance [15]-[17].

The contribution of this paper is an approach that exploits the properties of SUM regulatory functions in order to determine the number of the equilibrium points present in the genetic network and their location. The output of this approach is a collection of hypercubes that progressively shrink and converge to the sought set of equilibrium points. Some numerical examples illustrate and validate the proposed solution, and also show various cases where existing methods fail to provide the sought equilibrium points..

The paper is organized as follows. Section II provides some preliminaries about genetic networks with SUM regulatory functions. Section III introduces the proposed solution. Section IV illustrates the numerical results. Lastly, Section V provides some concluding discussions and future directions.

\section{PROBLEM FORMULATION}

First of all, let us introduce the notation used throughout the paper:

- $\mathbb{R}$ : real numbers set;

- $\mathbb{R}_{+}:\{x \in \mathbb{R}: x \geq 0\}$;

- $0_{n}$ : null vector of size $n \times 1$;

- $X^{\prime}$ : transpose of vector/matrix $X$;

- TF: transcription factor.

The genetic regulatory networks considered in this paper are described by differential equation models, and hence can 
be written according to

$$
\left\{\begin{aligned}
\dot{m}_{i}(t) & =-a_{i} m_{i}(t)+b_{i}\left(p_{1}(t), \ldots, p_{n}(t)\right) \\
\dot{p}_{i}(t) & =-c_{i} p_{i}(t)+d_{i} m_{i}(t) \\
i & =1, \ldots, n
\end{aligned}\right.
$$

where $m_{i}(t), p_{i}(t) \in \mathbb{R}_{+}$are the concentrations of mRNA and protein of the $i$-th gene, $a_{i}, c_{i} \in \mathbb{R}_{+}$are the degradation rates, and $d_{i} \in \mathbb{R}_{+}$expresses the effect of $m_{i}(t)$ on $p_{i}(t)$.

The function $b_{i}\left(p_{1}(t), \ldots, p_{n}(t)\right)$ is the regulatory function of the $i$-th gene, which is generally a nonlinear function of the variables $p_{1}(t), \ldots, p_{n}(t)$ satisfying the following properties. First,

$$
b_{i}\left(p_{1}(t), \ldots, p_{n}(t)\right) \in \mathbb{R}_{+} \forall p_{1}(t), \ldots, p_{n}(t) \in \mathbb{R}_{+}
$$

i.e. $b_{i}\left(p_{1}(t), \ldots, p_{n}(t)\right)$ is non-negative. Second,

$$
\begin{gathered}
\exists \delta_{1} \in \mathbb{R}: \max _{i=1, \ldots, n}\left|p_{i}(t)\right|<\delta_{1} \\
\Downarrow \\
\exists \delta_{2} \in \mathbb{R}: \quad b_{i}\left(p_{1}(t), \ldots, p_{n}(t)\right)<\delta_{2}
\end{gathered}
$$

i.e. $b_{i}\left(p_{1}(t), \ldots, p_{n}(t)\right)$ is bounded whenever $p_{1}(t), \ldots, p_{n}(t)$ are bounded. Third,

$$
\exists k_{j} \in\{-1,1\}: k_{j} \frac{d b_{i}\left(p_{1}(t), \ldots, p_{n}(t)\right)}{d p_{j}} \geq 0 \forall p_{j}(t) \in \mathbb{R}_{+}
$$

i.e. $b_{i}\left(p_{1}(t), \ldots, p_{n}(t)\right)$ is either monotonically increasing or monotonically decreasing with $p_{j}(t)$ for all fixed $p_{1}(t), \ldots, p_{j-1}(t), p_{j+1}(t), \ldots, p_{n}(t)$ for all $j=1, \ldots, n$.

Genetic networks with SUM regulatory functions own further properties, specifically the function $b_{i}\left(p_{1}(t), \ldots, p_{n}(t)\right)$ is expressed as the sum of functions of a single variable, i.e.

$$
b_{i}\left(p_{1}(t), \ldots, p_{n}(t)\right)=\sum_{j=1}^{n} \alpha_{i, j} b_{i, j}\left(p_{j}(t)\right)
$$

where $\alpha_{i, j} \in \mathbb{R}_{+}$is the contribution of TF $j$ to the transcriptional rate for gene $i$, and $b_{i, j}: \mathbb{R}_{+} \rightarrow \mathbb{R}_{+}$is a monotonic function. Each function $b_{i, j}\left(p_{j}(t)\right)$ in (5) is typically expressed as

$$
b_{i, j}\left(p_{j}(t)\right)= \begin{cases}f\left(p_{j}(t)\right) & \text { if TF } j \text { is an activator } \\ & \text { of gene } i \\ 1-f\left(p_{j}(t)\right) & \text { if TF } j \text { is a repressor } \\ & \text { of gene } i \\ 0 & \text { otherwise }\end{cases}
$$

where the function $f\left(p_{j}(t)\right)$ is a saturation function. For saturation function we mean a function satisfying the following properties:

1) $f: \mathbb{R}_{+} \rightarrow[0,1]$

2) $\frac{d f\left(p_{j}(t)\right)}{d p_{j}(t)}>0$ for all $p_{j}(t) \in \mathbb{R}_{+}$;

3) $f(0)=0$;

4) $\lim _{p_{j}(t) \rightarrow \infty} f\left(p_{j}(t)\right)=1$.

Hence, a saturation function is an increasing function between 0 and 1 defined for positive value of the variable. For instance, in the case of regulatory functions with Hill form, the function $f\left(p_{j}(t)\right)$ is given by

$$
f\left(p_{j}(t)\right)=\frac{p_{j}(t)^{H}}{\beta^{H}+p_{j}(t)^{H}}
$$

where $\beta \in \mathbb{R}_{+}$and $H$ is an integer known as Hill coefficient.

In order to describe the results of this paper in a more compact form, we introduce a matrix version of the model (1) according to

$$
\left\{\begin{aligned}
\dot{m}(t) & =A m(t)+R g(p(t))+r \\
\dot{p}(t) & =C p(t)+D m(t)
\end{aligned}\right.
$$

where

$$
\begin{aligned}
m(t) & =\left(m_{1}(t), \ldots, m_{n}(t)\right)^{\prime} \in \mathbb{R}^{n} \\
p(t) & =\left(p_{1}(t), \ldots, p_{n}(t)\right)^{\prime} \in \mathbb{R}^{n}
\end{aligned}
$$

are vectors containing the concentrations of mRNA and protein,

$$
\begin{aligned}
& A=\operatorname{diag}\left(-a_{1}, \ldots,-a_{n}\right) \in \mathbb{R}^{n \times n} \\
& C=\operatorname{diag}\left(-c_{1}, \ldots,-c_{n}\right) \in \mathbb{R}^{n \times n} \\
& D=\operatorname{diag}\left(d_{1}, \ldots, d_{n}\right) \in \mathbb{R}^{n \times n}
\end{aligned}
$$

are matrices containing the decay rates (matrices $A$ and $C$ ) and expressing the effect of $m(t)$ on $p(t)$ (matrix $D$ ), $R \in \mathbb{R}^{n \times n}$ and $r \in \mathbb{R}_{+}^{n}$ are defined as

$$
\begin{gathered}
R_{i, j}= \begin{cases}\alpha_{i, j} & \text { if } \mathrm{TF} j \text { is an activator of gene } i \\
-\alpha_{i, j} & \text { if } \mathrm{TF} j \text { is a repressor of gene } i \\
0 & \text { otherwise }\end{cases} \\
r_{i}=-\sum_{j: R_{i, j}<0} R_{i, j},
\end{gathered}
$$

and $g: \mathbb{R}_{+}^{n} \rightarrow[0,1]^{n}$ is defined as

$$
g(p(t))=\left(f\left(p_{1}(t)\right), \ldots, f\left(p_{n}(t)\right)\right)^{\prime} .
$$

The problem addressed in this paper consists of determining the equilibrium points of (8), i.e. the solutions of the system of nonlinear equations

$$
\left\{\begin{array}{l}
A m+R g(p)+r=0_{n} \\
C p+D m=0_{n} \\
m, p \in \mathbb{R}_{+}^{n}
\end{array}\right.
$$

Before proceeding, let us observe that the $m$-component of any solution of (14) is related to its $p$-component by the relationship $C p+D m=0_{n}$ where $C, D$ are nonsingular diagonal matrices with $C$ negative definite. This means that (14) can be equivalently rewritten as

$$
\left\{\begin{array}{l}
-A D^{-1} C p+R g(p)+r=0_{n} \\
m=-D^{-1} C p \\
p \in \mathbb{R}_{+}^{n}
\end{array}\right.
$$

Therefore, in the sequel we will focus on the computation of the set of vectors $p$ fulfilling (15), that we indicate as

$$
\mathcal{E}=\left\{p \in \mathbb{R}_{+}^{n}:-A D^{-1} C p+b(p)=0_{n}\right\} .
$$


Also, let us observe that genetic networks with SUM regulatory functions can be also modeled with time-delays. Clearly, the computation of the equilibrium points of such a system can be reformulated as in (14) because the equilibrium points are constant solutions of the system of differential equations, and hence terms like $m\left(t-\tau_{m}\right)$ and $p\left(t-\tau_{p}\right)$ coincide with the terms $m(t)$ and $p(t)$ once the steady-state has been reached.

Lastly, genetic networks with SUM regulatory functions can be also modeled as stochastic systems, for example by adding a stochastic process after $r$ in (8). In these systems there are no equilibrium points in the classic sense since the input is a non-constant function of the time and hence the steady-state is never reached. Instead, there are equilibrium points corresponding to particular constant values of the stochastic process, such as its mean value, which can be readily considered by introducing such values in (14).

\section{Characterizing The EQUilibrium POINTS}

In this section we describe the proposed approach for solving (14), in particular for computing the set $\mathcal{E}$ in (16).

Let us start by defining the following function:

$$
\mathcal{A}(\mathcal{H})=\left\{p \in \mathbb{R}_{+}^{n}: p_{i} \in\left[q_{i,-}, q_{i,+}\right] \forall i=1, \ldots, n\right\} .
$$

In (17), $\mathcal{H}$ is the rectangle defined as

$$
\mathcal{H}=\left\{p \in \mathbb{R}_{+}^{n}: p_{i} \in\left[p_{i,-}, p_{i,+}\right] \forall i=1, \ldots, n\right\}
$$

for some $p_{1,-}, p_{1,+}, \ldots, p_{n,-}, p_{n,+} \in \mathbb{R}_{+}$. The quantities $q_{1,-}, q_{1,+}, \ldots, q_{n,-}, q_{n,+} \in \mathbb{R}_{+}$are given by

$$
\begin{aligned}
& q_{i,-}=\max \left\{p_{i,-}, r_{i}+s_{i,-}\right\} \\
& q_{i,+}=\min \left\{p_{i,+}, r_{i}+s_{i,+}\right\}
\end{aligned}
$$

where $s_{i,-}, s_{i,+}$ are defined according to

$$
\begin{aligned}
s_{i,-} & =\min _{p \in \operatorname{ver}(\mathcal{H})} \xi_{i}(p) \\
s_{i,+} & =\max _{p \in \operatorname{ver}(\mathcal{H})} \xi_{i}(p),
\end{aligned}
$$

$\operatorname{ver}(\mathcal{H})$ is the set of vertices of $\mathcal{H}$, i.e.

$$
\operatorname{ver}(\mathcal{H})=\left\{p: p_{i} \in\left\{p_{i,-}, p_{i,+}\right\} \forall i=1, \ldots, n\right\},
$$

$\xi_{i}(p)$ is the function

$$
\xi_{i}(p)=\frac{d_{i}}{a_{i} c_{i}} r_{i} g(p),
$$

and $r_{i}$ is the $i$-th row of $R$, i.e.

$$
r_{i}=\left(R_{i, 1}, \ldots, R_{i, n}\right) .
$$

Let us observe that $\mathcal{A}(\mathcal{H})$ is a rectangle, i.e. the function $\mathcal{A}(\mathcal{H})$ transforms the rectangle $\mathcal{H}$ in another rectangle. Let us also observe that the set $\operatorname{ver}(\mathcal{H})$ is finite being the collection of the vertices of the rectangle $\mathcal{H}$. This means that the computation of $s_{1,-}, s_{1,+}, \ldots, s_{n,-}, s_{n,+}$, and hence the computation of $\mathcal{A}(\mathcal{H})$, just requires the evaluation of the function $\xi_{i}(p)$ at a finite number of points.

The function $\mathcal{A}(\mathcal{H})$ owns some useful properties. First,

$$
\mathcal{A}(\mathcal{H}) \subseteq \mathcal{H}
$$

since one has that $q_{i,-}$ is always greater than or equal to $p_{i,-}$, and similarly $q_{i,+}$ is always lesser than or equal to $p_{i,+}$. Second,

$$
p \in \mathcal{H} \cap \mathcal{E} \Rightarrow p \in \mathcal{A}(\mathcal{H})
$$

because $\xi_{i}(p)$ is a linear function of $g(p)$, each entry of $g(p)$ is a monotonic function of an entry of $p$, and $\xi_{i}(p)=-r_{i}+p_{i}$ if $p \in \mathcal{E}$. Third,

$$
\mathcal{H} \cap \mathcal{A}(\mathcal{H})=\emptyset \Rightarrow \mathcal{H} \cap \mathcal{E}=\emptyset
$$

because, if one supposes for contradiction that $\mathcal{H} \cap \mathcal{E} \neq \emptyset$, then it would follow from (26)-(27) that $\mathcal{H} \cap \mathcal{A}(\mathcal{H}) \neq \emptyset$, hence contradicting the assumption in (28).

Next, we define the function $\mathcal{B}(\mathcal{H})$ as follows:

$$
\mathcal{B}(\mathcal{H})= \begin{cases}\emptyset & \text { if } \mathcal{H} \cap \mathcal{A}(\mathcal{H})=\emptyset \\ \mathcal{A}(\mathcal{H}) & \text { if } \mathcal{A}(\mathcal{H}) \text { is a point } \\ \mathcal{H} & \text { if } \mathcal{H}=\mathcal{A}(\mathcal{H}) \\ \mathcal{B}(\mathcal{A}(\mathcal{H})) & \text { otherwise }\end{cases}
$$

Let us observe that the function $\mathcal{B}(\mathcal{H})$ transforms the rectangle $\mathcal{H}$ into either a rectangle, a point, or the empty set. This is achieved by consecutive uses of the function $\mathcal{A}(\mathcal{H})$. The function $\mathcal{B}(\mathcal{H})$ owns some useful properties. First,

$$
\mathcal{B}(\mathcal{H}) \subseteq \mathcal{H}
$$

because (26) ensures that the output of the function $\mathcal{A}(\mathcal{H})$ is included in its input. Second,

$$
p \in \mathcal{H} \cap \mathcal{E} \Rightarrow p \in \mathcal{B}(\mathcal{H})
$$

because (27) ensures that no point lying inside the set $\mathcal{H} \cap \mathcal{E}$ can be lost by $\mathcal{A}(\mathcal{H})$.

Finally, we define the sought approach for computing the equilibrium points of the genetic regulatory network (8). This approach is represented by the following function $\mathcal{C}(\mathcal{H})$ :

$$
\mathcal{C}(\mathcal{H})= \begin{cases}\mathcal{B}(\mathcal{H}) & \text { if } \mathcal{B}(\mathcal{H}) \text { is either the } \\ \mathcal{C}\left(\mathcal{H}_{1}\right) \cup \ldots \cup \mathcal{C}\left(\mathcal{H}_{k}\right) & \text { empty set or a point } \\ \text { otherwise }\end{cases}
$$

where $\mathcal{H}_{1}, \ldots, \mathcal{H}_{k}$ are rectangles satisfying

$$
\mathcal{B}(\mathcal{H})=\bigcup_{i=1, \ldots, k} \mathcal{H}_{i}
$$

which are obtained by dividing the rectangle $\mathcal{B}(\mathcal{H})$ into smaller ones, for example by taking the middle point of each side of $\mathcal{B}(\mathcal{H})$ with nonzero length as partitioning point.

The computation of the equilibrium points of the genetic regulatory network (8) is obtained as follows:

$$
\hat{\mathcal{E}}=\mathcal{C}\left(\mathbb{R}_{+}^{n}\right)
$$

which means that the positive octant $\mathbb{R}_{+}^{n}$ is used as initial rectangle $\mathcal{H}$. In fact, $\mathbb{R}_{+}^{n}$ is clearly guaranteed to contain all solutions of (15).

Let us observe that the function $\mathcal{C}(\mathcal{H})$ is obtained through consecutive uses of the function $\mathcal{B}(\mathcal{H})$. The function $\mathcal{C}\left(\mathbb{R}_{+}^{n}\right)$ owns some useful properties. First, the positive octant $\mathbb{R}_{+}^{n}$ is 
progressively shrunk without losing any point of $\mathcal{E}$. In fact, $\mathcal{B}(\cdot)$ preserves any vector in $\mathcal{E}$ according to (31), moreover from (30) one has that the set progressively constructed cannot increase. Second, the output of $\mathcal{C}\left(\mathbb{R}_{+}^{n}\right)$ coincides with the sought set of equilibrium points, i.e.

$$
\hat{\mathcal{E}}=\mathcal{E} .
$$

In fact, no portion of $\mathbb{R}_{+}^{n}$ is lost in the division of each rectangle $\mathcal{B}(\mathcal{H})$ into the rectangles $\mathcal{H}_{1}, \ldots, \mathcal{H}_{k}$, and hence (35) holds due to (31).

In conclusion, $\mathcal{C}\left(\mathbb{R}_{+}^{n}\right)$ passes the initial rectangle $\mathbb{R}_{+}^{n}$ to the function $\mathcal{B}(\mathcal{H})$. If the output of this function is either the empty set or a point, then the search stops as it is guaranteed that there are no equilibrium points inside the considered rectangle. Otherwise, the output is a rectangle, which is then divided into smaller ones, and then passed to the function $\mathcal{B}(\mathcal{H})$ itself.

\section{ILLUSTRATIVE EXAMPLES}

This section presents some numerical examples that illustrate the main steps of the proposed approach. For conciseness, we report only the $p$-component of each equilibrium point, being the $m$-component directly given by $m=D^{-1} C p$ according to (15). The computational time on a standard personal is less than one minute for all examples.

\section{A. Example 1}

Let us start by considering the genetic network with SUM regulatory functions

$$
\left\{\begin{aligned}
\dot{m}_{1} & =-0.8 m_{1}+0.8 f\left(p_{1}\right)+1-f\left(p_{2}\right) \\
\dot{m}_{2} & =-0.4 m_{2}+1.5\left(1-f\left(p_{1}\right)\right) \\
\dot{p}_{1} & =-1.1 p_{1}+m_{1} \\
\dot{p}_{2} & =-1.8 p_{2}+m_{2}
\end{aligned}\right.
$$

where $f(\cdot)$ is the saturation function given by

$$
f\left(p_{i}\right)=\frac{2}{\pi} \arctan \left(p_{i}^{2}\right) .
$$

The problem consists of determining the equilibrium points of this system, i.e. the solutions of the system of nonlinear equations (14).

First of all, let us rewrite this system as in (8) with

$$
\begin{aligned}
A & =\left(\begin{array}{cc}
-0.8 & 0 \\
0 & -0.4
\end{array}\right) \\
C & =\left(\begin{array}{cc}
-1.1 & 0 \\
0 & -1.8
\end{array}\right) \\
D & =\left(\begin{array}{cc}
1 & 0 \\
0 & 1
\end{array}\right) \\
R & =\left(\begin{array}{cc}
0.8 & -1 \\
-1.5 & 0
\end{array}\right) \\
r & =\left(\begin{array}{c}
1 \\
1.5
\end{array}\right) .
\end{aligned}
$$

Then, let us use the function $\mathcal{C}\left(\mathbb{R}_{+}^{n}\right)$ in (32). The positive octant $\mathbb{R}_{2}^{+}$is initially shrunk to the rectangle shown in Figure 1a. Then, the rectangle previously found is divided in four equal rectangles, one of which is shown in Figure $1 b$, another one shrinks to the equilibrium point shown in the same figure, and the other two are discarded via the function $\mathcal{B}(\cdot)$. Proceeding, another equilibrium point is found as shown in Figure 1c, and only one rectangle is left. Finally, the last equilibrium point is found as shown in Figure 1d. We hence conclude that the set $\mathcal{E}$ in (16) is given by

$$
\mathcal{E}=\left\{\left(\begin{array}{l}
1.726 \\
0.430
\end{array}\right),\left(\begin{array}{l}
1.014 \\
1.024
\end{array}\right),\left(\begin{array}{l}
0.193 \\
2.034
\end{array}\right)\right\}
$$

Now, we attempt to solve the same problem by using the function "solve" of Matlab for solving systems of nonlinear equations, and we obtain one equilibrium point only, which is found by using iterative techniques such as Newton's method. Indeed, it is worth to remark that no existing method guarantees to find all solutions of a system of nonlinear equations.

\section{B. Example 2}

Here we consider a real biological example, specifically the genetic regulatory network in the repressilator investigated in Escherichia coli [18]:

$$
\left\{\begin{array}{l}
\dot{m}_{i}=-m_{i}+\alpha^{r e p}\left(1-f\left(p_{j}\right)\right) \\
\dot{p}_{i}=-\beta^{r e p}\left(p_{i}-m_{i}\right) \\
i=\text { lacl, tet } R, c l \\
j=\text { cl, lacl, tet } R
\end{array}\right.
$$

where the saturation function is the Hill function

$$
f\left(p_{i}\right)=\frac{p_{i}^{2}}{1+p_{i}^{2}}
$$

and $\alpha^{r e p}, \beta^{r e p} \in \mathbb{R}_{+}$are positive constants.

We select the plausible values $\alpha^{r e p}=10$ and $\beta^{r e p}=1$, for which the mRNA and protein concentrations of the repressilator oscillate. By using the function $\mathcal{C}\left(\mathbb{R}_{+}^{n}\right)$ in (32) we find that the set $\mathcal{E}$ in (16) is given by

$$
\mathcal{E}=\left\{\left(\begin{array}{l}
2.000 \\
2.000 \\
2.000
\end{array}\right)\right\}
$$

\section{Example 3}

In this example we consider a genetic regulatory network with 16 state variables, specifically

$$
\left\{\begin{array}{l}
\dot{m}_{1}(t)=-2 m_{1}(t)+z_{1}(p) \\
\dot{m}_{2}(t)=-m_{2}(t)+z_{2}(p) \\
\dot{m}_{3}(t)=-0.8 m_{3}(t)+z_{3}(p) \\
\dot{m}_{4}(t)=-1.2 m_{4}(t)+z_{4}(p) \\
\dot{m}_{5}(t)=-1.5 m_{5}(t)+z_{5}(p) \\
\dot{m}_{6}(t)=-0.9 m_{6}(t)+z_{6}(p) \\
\dot{m}_{7}(t)=-1.5 m_{7}(t)+z_{7}(p) \\
\dot{m}_{8}(t)=-1.2 m_{8}(t)+z_{8}(p) \\
\dot{p}_{i}(t)=-p_{i}(t)+m_{i}(t) \quad \forall i=1, \ldots, 8
\end{array}\right.
$$




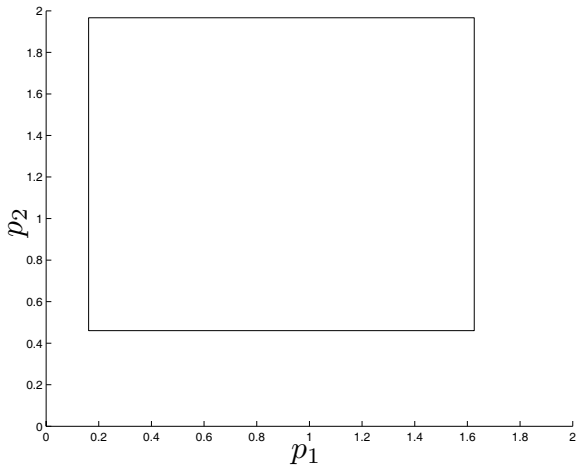

(a)

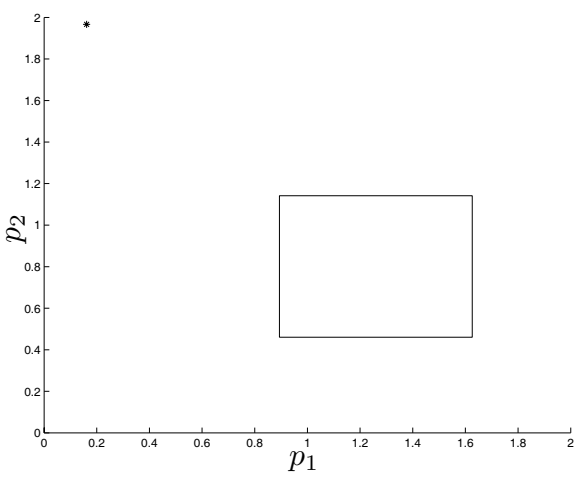

(b)

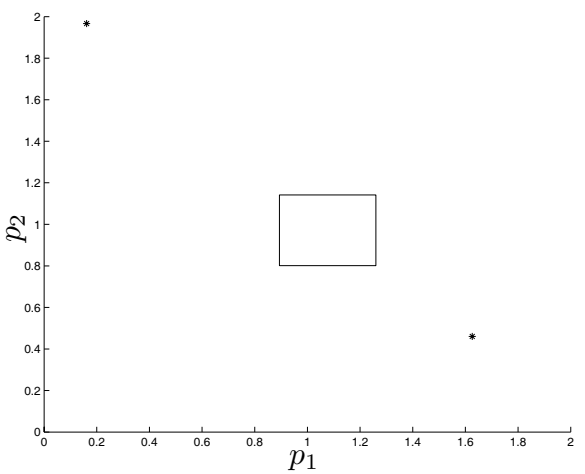

(c)

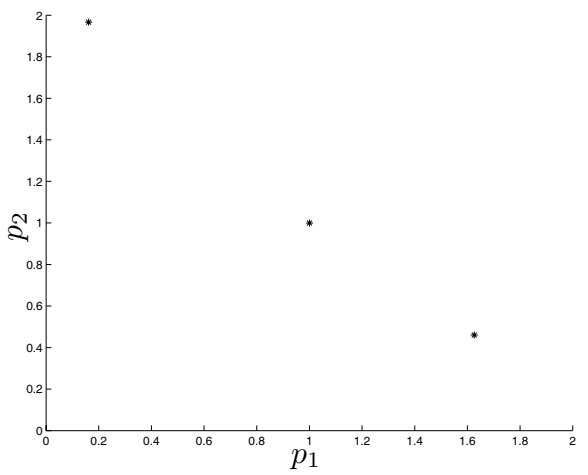

(d)

Fig. 1. Example 1: steps of the proposed approach. (a): $\mathbb{R}_{+}^{2}$ is shrunk to a rectangle. (b): an equilibrium point is found (denoted by the "*” mark). (c): another equilibrium point is found. (d): the last equilibrium point is found. where the functions $z_{1}(p), \ldots, z_{8}(p)$ are given by

$$
\begin{aligned}
z_{1}(p)= & 0.2 f\left(p_{1}\right)+0.3\left(1-f\left(p_{2}\right)\right)+0.2 f\left(p_{3}\right) \\
& +0.2 f\left(p_{4}\right)+0.1\left(1-f\left(p_{5}\right)\right)+0.2\left(1-f\left(p_{6}\right)\right) \\
& +0.3 f\left(p_{7}\right)+0.3 f\left(p_{8}\right) \\
z_{2}(p)= & 0.4 f\left(p_{1}\right)+0.1 f\left(p_{2}\right)+0.5\left(1-f\left(p_{3}\right)\right) \\
& +0.5\left(1-f\left(p_{4}\right)\right)+0.1\left(1-f\left(p_{5}\right)\right)+0.2 f\left(p_{6}\right) \\
& +0.2\left(1-f\left(p_{7}\right)\right)+0.2\left(1-f\left(p_{8}\right)\right) \\
z_{3}(p)= & 0.4\left(1-f\left(p_{1}\right)\right)+0.5 f\left(p_{2}\right)+0.1\left(1-f\left(p_{3}\right)\right) \\
& +0.2\left(1-f\left(p_{4}\right)\right)+0.3 f\left(p_{5}\right)+0.2\left(1-f\left(p_{6}\right)\right) \\
& +0.3 f\left(p_{7}\right)+0.5 f\left(p_{8}\right) \\
z_{4}(p)= & 0.4 f\left(p_{1}\right)+0.1\left(1-f\left(p_{2}\right)\right)+0.4 f\left(p_{3}\right) \\
& +0.1\left(1-f\left(p_{4}\right)\right)+0.3 f\left(p_{5}\right)+0.3\left(1-f\left(p_{6}\right)\right) \\
& +0.2 f\left(p_{7}\right)+0.3\left(1-f\left(p_{8}\right)\right) \\
z_{5}(p)= & 0.1 f\left(p_{1}\right)+0.3 f\left(p_{2}\right)+0.2 f\left(p_{3}\right) \\
& +0.4\left(1-f\left(p_{4}\right)\right)+0.2 f\left(p_{5}\right)+0.4\left(1-f\left(p_{6}\right)\right) \\
& +0.4 f\left(p_{7}\right)+0.4 f\left(p_{8}\right) \\
z_{6}(p)= & 0.4\left(1-f\left(p_{1}\right)\right)+0.4\left(1-f\left(p_{2}\right)\right)+0.3 f\left(p_{3}\right) \\
& +0.3 f\left(p_{4}\right)+0.1\left(1-f\left(p_{5}\right)\right)+0.1 f\left(p_{6}\right) \\
& +0.5 f\left(p_{7}\right)+0.2\left(1-f\left(p_{8}\right)\right) \\
z_{7}(p)= & 0.2\left(1-f\left(p_{1}\right)\right)+0.1\left(1-f\left(p_{2}\right)\right)+0.2 f\left(p_{3}\right) \\
& +0.2 f\left(p_{4}\right)+0.1\left(1-f\left(p_{5}\right)\right)+0.5 f\left(p_{6}\right) \\
& +0.1\left(1-f\left(p_{7}\right)\right)+0.3\left(1-f\left(p_{8}\right)\right) \\
z_{8}(p)= & 0.5 f\left(p_{1}\right)+0.4 f\left(p_{2}\right)+0.3\left(1-f\left(p_{3}\right)\right) \\
& +0.2\left(1-f\left(p_{4}\right)\right)+0.4 f\left(p_{5}\right)+0.2\left(1-f\left(p_{6}\right)\right) \\
& +0.4\left(1-f\left(p_{7}\right)\right)+0.2\left(1-f\left(p_{8}\right)\right) \\
& +00
\end{aligned}
$$

and the saturation function is the Hill function

$$
f\left(p_{i}\right)=\frac{p_{i}^{2}}{1+p_{i}^{2}} .
$$

Analogously to the previous examples, by using the function $\mathcal{C}\left(\mathbb{R}_{+}^{n}\right)$ in (32) we conclude that there are three equilibrium points, in particular the set $\mathcal{E}$ in (16) is given by

$$
\mathcal{E}=\left\{\left(\begin{array}{l}
0.375 \\
1.014 \\
1.564 \\
0.737 \\
0.718 \\
1.423 \\
0.715 \\
0.883
\end{array}\right)\right\} .
$$

It is interesting to observe that also in this case we cannot reach the same conclusion by using existing methods, though the equations in the system (14) are rational in this case (and, hence, (14) can be equivalently rewritten via polynomial equations). In fact, iterative techniques such as homotopy methods do not guarantee to find all solutions, and hence they cannot allow one to conclude that there is only one equilibrium point. Then, analytical techniques such as the resultants method provide the sought solutions as roots of a one-variable polynomial obtained via variables elimination, but the degree of such a polynomial can be up to the degree of the polynomial equations to the power of the number of variables in the system (14), which is given in the present 
case by $16^{8} \approx 4.3 \cdot 10^{9}$ : in fact, the degree of the polynomial equations is 16 since the equations in (14) are sums of eight rational functions of degree 2 , and the number of variables is given by $n$ which is equal to 8 .

\section{CONCLUSION}

We have considered the problem of characterizing the equilibrium points of genetic networks with SUM regulatory functions. For this problem we have proposed an approach which guarantees to find all sought equilibria and which is based on the progressive deformation of the positive octant. This approach exploits the properties of SUM regulatory functions and compares favorably with existing methods, which very often fail as shown via some numerical examples. It is hence believed that the proposed approach may be a very useful tool for researchers in the area of genetic regulatory networks. Future work will consider the extension of this approach to other classes of genetic regulatory networks.

\section{ACKNOWLEDGEMENT}

The author would like to thank the Editors and the Reviewers for their useful and encouraging comments.

\section{REFERENCES}

[1] C.-H. Yuh, H. Bolouri, and E. H. Davidson, "Genomic cis-regulatory logic: Experimental and computational analysis of a sea urchin gene," Science, vol. 279, pp. 1896-1902, 1998.

[2] P. D'haeseleer, X. Wen, S. Fuhrman, and R. Somogyi, "Mining the gene expression matrix: Inferring gene relationships from large scale gene expression data," in Information Processing in Cells and Tissues, R. C. Paton and M. Holcombe, Eds. Plenum Publishing, 1998.

[3] P. D'haeseleer, S. Liang, and R. Somogyi, "Genetic network inference: From co-expression clustering to reverse engineering," Bioinformatics, vol. 16 , no. 8, pp. 707-726, 2000.

[4] C. Li, L. Chen, and K. Aihara, "A systems biology perspective on signal processing in genetic network motifs," IEEE Signal Processing Magazine, vol. 221, no. 3, pp. 136-142, 2007.

[5] I. A. Maraziotis, A. Dragomir, and A. Bezerianos, "Gene networks reconstruction and time-series prediction from microarray data using recurrent neural fuzzy networks," IET Systems and Biology, vol. 1, no. 1, pp. 41-50, 2007.

[6] P. Smolen, D. A. Baxter, and J. H. Byrne, "Mathematical modeling of gene networks," Neuron, vol. 26, no. 3, pp. 567-580, 2000.

[7] J. M. Bower and H. Bolouri, Eds., Computational Modeling of Genetic and Biochemical Networks, ser. Computational Molecular Biology. MIT Press, 2001.

[8] H. D. Jong, "Modeling and simulation of genetic regulatory systems: A literature review," Journal of Computation Biology, vol. 9, pp. 67-103, 2002.

[9] P. D'haeseleer, S. Liang, and R. Somogyi, "Gene expression data analysis and modeling," in Pacific Symposium on Biocomputing, Hawaii, USA, 1999.

[10] J. Aracena, S. B. Lamine, M. A. Mermet, O. Cohen, and J. Demongeot, "Mathematical modeling in genetic networks: Relationships between the genetic expression and both chromosomic breakage and positive circuits," IEEE Transactions on Systems, Man, and CyberneticsPart b: Cybernetics, vol. 33, no. 5, pp. 825-834, 2003.

[11] G. Chesi, A. Garulli, A. Tesi, and A. Vicino, "Characterizing the solution set of polynomial systems in terms of homogeneous forms: an LMI approach," Int. Journal of Robust and Nonlinear Control, vol. 13, no. 13, pp. 1239-1257, 2003.

[12] T. Mora, Solving Polynomial Equation Systems II. Cambridge University Press, 2005.

[13] J. Nocedal and S. Wright, Numerical Optimization, ser. Springer Series in Operations Research and Financial Engineering. Springer, 2006.
[14] G. Chesi, "Optimal representation matrices for solving polynomial systems via LMI," Int. Journal of Pure and Applied Mathematics, vol. 45 no. 3 , pp. 397-412, 2008

[15] C. Li, L. Chen, and K. Aihara, "Stability of genetic networks with sum regulatory logic: Lure system and LMI approach," IEEE Trans. on Circuits and Systems I, vol. 53, no. 11, pp. 2451-2458, 2006.

[16] —, "Stochastic stability of genetic networks with disturbance attenuation," IEEE Transactions on Circuits and Systems II, vol. 54, no. 10, pp. 892-896, 2007.

[17] G. Chesi and Y. S. Hung, "Stability analysis of uncertain genetic SUM regulatory networks," Automatica, vol. 44, no. 9, pp. 2298-2305, 2008.

[18] M. B. Elowitz and S. Leibler, "A synthetic oscillatory network of transcriptional regulators," Nature, vol. 403, pp. 335-338, 2000. 